\title{
A Thienopyridazinone-Based Melanin-Concentrating Hormone Receptor 1 Antagonist With Potent In Vivo Anorectic Properties
}

Brian Dyck, ${ }^{1}$ Stacy Markison, Liren Zhao, Junko Tamiya, Jonathan Grey, Martin W. Rowbottom, Mingzhu Zhang, Troy Vickers, Katie Sorensen, Christi Norton, Jenny Wen, Christopher E. Heise, John Saunders, Paul Conlon, Ajay Madan, David Schwarz, Val S. Goodfellow.

Neurocrine Biosciences Inc., 12790 El Camino Real, San Diego, CA 92130.

\section{Supporting Information}

Table of Contents

$\begin{array}{lc}\text { Chemistry Procedures } & \text { S2 } \\ \text { Table of Elemental Analysis Data } & \text { S13 } \\ \text { In Vitro Binding } & \text { S13 } \\ \text { Feeding Studies } & \text { S14 } \\ \text { Human Liver Microsome Clearance } & \text { S14 } \\ \text { Rat Pharmacokinetic Studies } & \text { S15 } \\ \text { References } & \text { S15 }\end{array}$

${ }^{1}$ Corresponding author. Fax +1-858-617-7619; e-mail: bdyck@neurocrine.com 
Chemistry. General methods. ${ }^{1}$ H NMR spectra were obtained with a Varian $300 \mathrm{MHz}$ spectrometer. The chemical shifts are reported in parts per million $(\delta)$ downfield using TMS as the internal standard and $\mathrm{CDCl}_{3}$ as the solvent except where indicated. High resolution ESI-TOF mass spectra were obtained from The Scripps Center for Mass Spectrometry, San Diego, CA. All final compounds were analyzed on an Agilent 1100 LCMS by using either a Phenomenex Synergi MaxRP 50 × 2 mm reverse phase column with a 5-95\% acetonitrile/water gradient containing $0.025 \%$ TFA (method 1 ) or using a Berger Pyridine 4.6 x 150 mm supercritical fluid chromatography column and a 10-55\% methanol, $1.5 \%$ water and $0.025 \%$ ethanesulfonic acid gradient (method 2 ). Final compounds were shown to be at least $95 \%$ pure based on both UV wavelengths $(220 \mathrm{nM}$, $254 \mathrm{nM}$ ) under these conditions. All commercially available reagents were used without further purification. Hydrochloride salts of final compounds used for pharmacokinetic measurements were prepared by treating the free bases in DCM with excess $\mathrm{HCl}$ in ether, concentration of the mixture, and recrystallization as necessary. Methanesulfonic acid salts were prepared by addition of a single equivalent of the sulfonic acid from a stock solution, concentration, and recrystallization as necessary.

6-Bromo-2H-phthalazin-1-one (7). Bromophthalimide 5 (13.0 g, 57.8 mmol) was added in six portions over 30 minutes to a stirred suspension of zinc powder $(4.50 \mathrm{~g}, 69.2$ $\mathrm{mmol})$ and copper(II) sulfate pentahydrate $(0.060 \mathrm{~g}, 0.25 \mathrm{mmol})$ in aqueous sodium hydroxide $(2 \mathrm{M}, 71 \mathrm{~mL})$ at $0^{\circ} \mathrm{C}$ (ice-bath.) The mixture was stirred at $0^{\circ} \mathrm{C}$ for an additional 30 minutes, and at room temperature for $2.5 \mathrm{~h}$ to complete the reaction. After filtering, the reaction solution was neutralized to $\mathrm{pH} 7$ with $20 \%$ hydrochloric acid, diluted with $100 \mathrm{~mL}$ of ethanol, and then extracted with ethyl acetate. The extract was washed with brine, dried with $\mathrm{MgSO}_{4}$ and concentrated in vacuo to afford $12.5 \mathrm{~g} \mathrm{(95 \% )}$ of 6 as a yellow solid: LC-MS $210\left(\mathrm{MH}^{+}-\mathrm{H}_{2} \mathrm{O}\right)$. A suspension of this material and hydrazine $(367 \mathrm{~g}, 1.15 \mathrm{~mol})$ in $246 \mathrm{~mL}$ of water was heated at $95^{\circ} \mathrm{C}$ for $3 \mathrm{~h}$. The yellow solid that precipitated was filtered and washed with water. This crude material was triturated with hot ethyl acetate to afford $4.1 \mathrm{~g}$ (34\%) of 7 as a yellow solid: ${ }^{1} \mathrm{H}$ NMR (300 MHz, 1:1 $\left.\mathrm{CDCl}_{3} / \mathrm{DMSO}_{6}\right) \delta 8.26(\mathrm{~s}, 1 \mathrm{H}), 8.21(\mathrm{~s}, 1 \mathrm{H}), 8.15(\mathrm{~d}, \mathrm{~J}=2.1 \mathrm{~Hz}, 1 \mathrm{H})$, $8.10(\mathrm{~d}, \mathrm{~J}=8.7 \mathrm{~Hz}, 1 \mathrm{H}), 7.92(\mathrm{dd}, \mathrm{J}=8.6 \mathrm{~Hz}, 1 \mathrm{H})$; LC-MS $225\left(\mathrm{MH}^{+}\right)$. 
6-(4-Chlorophenyl)-2H-phthalazin-1-one (8a). Nitrogen was bubbled through a suspension of 7 (4.50 g, $20.0 \mathrm{mmol})$, 4-chlorophenylboronic acid (3.90 g, $25.0 \mathrm{mmol})$ and potassium carbonate $(5.50 \mathrm{~g}, 39.9 \mathrm{mmol})$ in $\mathrm{DMF}(300 \mathrm{~mL})$ and water $(30 \mathrm{~mL})$ for 10 minutes, and then [1,1'-bis(diphenylphosphino)-ferrocene]dichloropalladium(II) DCM complex (1.46 g, $2.00 \mathrm{mmol})$ was added. After the reaction mixture was heated at $80^{\circ} \mathrm{C}$ for $5 \mathrm{~h}$, cooled and filtered through Celite, it was partitioned between water $(250 \mathrm{~mL})$ and ethyl acetate $(400 \mathrm{~mL})$. The organic extract was washed with brine, dried with $\mathrm{MgSO}_{4}$ and concentrated in vacuo. The resulting oil was stirred in DCM (100 mL) and the resulting yellow solid was isolated by filtration. This material was purified by flash chromatography (elution with $0.2 \%$ ammonium hydroxide and $3 \%$ methanol in DCM) to afford $1.1 \mathrm{~g}(22 \%)$ of 8a: ${ }^{1} \mathrm{H}$ NMR (300 MHz, DMSO-d 6 ) $\delta 8.39$ (s, $\left.1 \mathrm{H}\right), 8.28-8.26$ (m, 2 H), $8.14(\mathrm{dd}, \mathrm{J}=8.4,1.8 \mathrm{~Hz}), 7.86(\mathrm{~d}, \mathrm{~J}=8.7 \mathrm{~Hz}, 2 \mathrm{H}), 7.61$ (d, J = 9.0 Hz, $2 \mathrm{H})$; LC-MS $257\left(\mathrm{MH}^{+}\right)$. The following pyridazinone derivatives $\mathbf{8}$ were prepared using the same procedure.

6-(4-Trifluoromethylphenyl)-2H-phthalazin-1-one (8b). ${ }^{1} \mathrm{H}$ NMR (300 MHz, DMSO$\left.\mathrm{d}_{6}\right) \delta 8.42(\mathrm{~s}, 1 \mathrm{H}), 8.32-8.29(\mathrm{~m}, 2 \mathrm{H}), 8.20(\mathrm{dd}, \mathrm{J}=8.4,1.8 \mathrm{~Hz}, 1 \mathrm{H}), 8.04(\mathrm{~d}, \mathrm{~J}=7.8 \mathrm{~Hz}$, $2 \mathrm{H}), 7.90$ (d, J = 8.4 Hz, $2 \mathrm{H})$; LC-MS $291\left(\mathrm{MH}^{+}\right)$.

6-(4-Methoxy-2-methylphenyl)-2H-phthalazin-1-one (8c). ${ }^{1} \mathrm{H}$ NMR (300 MHz, DMSO-d $\left._{6}\right) \delta 8.37(\mathrm{~s}, 1 \mathrm{H}), 8.22(\mathrm{~d}, \mathrm{~J}=5.4 \mathrm{~Hz}, 1 \mathrm{H}), 7.86(\mathrm{~d}, \mathrm{~J}=1.2 \mathrm{~Hz}, 1 \mathrm{H}), 7.78(\mathrm{dd}, \mathrm{J}$ = 8.1, $1.5 \mathrm{~Hz}, 1 \mathrm{H}), 7.23$ (d, J = 8.4 Hz, 1 H), 6.93-6.86 (m, $2 \mathrm{H}), 3.78$ (s, $3 \mathrm{H}), 2.24$ (s, 3 H); LC-MS $267\left(\mathrm{MH}^{+}\right)$.

\section{4-Chloro-2-(tetrahydropyran-2-yl)-5-(4-trifluoromethylphenylethynyl)-2H-} pyridazin-3-one (11a). Compound 10 ${ }^{1,2}(2.73 \mathrm{~g}, 11.8 \mathrm{mmol})$ and TEA $(2.5 \mathrm{~mL}, 18$ mmol) were dissolved in DCM $(80 \mathrm{~mL})$ and cooled in an ice bath. Trifluoromethanesulfonic anhydride $(2.4 \mathrm{~mL}, 14 \mathrm{mmol})$ was added over five minutes, and the mixture was stirred at $0^{\circ} \mathrm{C}$ for 30 minutes. The mixture was poured into $0.5 \mathrm{M}$ hydrochloric acid $(50 \mathrm{~mL})$ and extracted three times with DCM (50 mL.) The combined 
extracts were washed with $1 \%$ aqueous sodium bicarbonate $(50 \mathrm{~mL})$ and aqueous sodium chloride $(30 \mathrm{~mL})$, dried $\left(\mathrm{MgSO}_{4}\right)$, and concentrated under vacuum to afford $3.84 \mathrm{~g}(90 \%)$ of the sulfonate as a brown oil. A portion of this material $(1.48 \mathrm{~g}, 4.08 \mathrm{mmol})$ was dissolved in THF (22 mL.) Dichlorobis(triphenylphosphine)palladium(II) (84 mg, 0.12 $\mathrm{mmol})$, copper(I) iodide (226 mg, $1.19 \mathrm{mmol})$ and tetra- $n$-butylammonium iodide $(4.51 \mathrm{~g}$, $12.2 \mathrm{mmol}$ ) were added and the mixture was stirred for 10 seconds prior to the addition of TEA (1.55 mL, $11.1 \mathrm{mmol})$ and 4-(trifluoromethyl)phenylacetylene (0.67 mL, 4.1 mmol.) The mixture was stirred for $3 \mathrm{~h}$, ethyl acetate $(44 \mathrm{~mL})$ was added, and the mixture was filtered through a pad of Celite. The mixture was concentrated under vacuum and the residue was purified by flash chromatography (elution with $20 \%$ ethyl acetate in hexaneS) to afford $467 \mathrm{mg}(82 \%)$ of 11a as a white powder: ${ }^{1} \mathrm{H}$ NMR (300 $\left.\mathrm{MHz}, \mathrm{CDCl}_{3}\right) \delta 7.86(\mathrm{~s}, 1 \mathrm{H}), 7.72(\mathrm{~d}, \mathrm{~J}=5.4 \mathrm{~Hz}, 2 \mathrm{H}), 7.66(\mathrm{~d}, \mathrm{~J}=5.4 \mathrm{~Hz}, 2 \mathrm{H}), 6.06$ $(\mathrm{dd}, \mathrm{J}=11,4.8 \mathrm{~Hz}, 1 \mathrm{H}), 3.76(\mathrm{dt}, \mathrm{J}=12,2.7 \mathrm{~Hz}, 1 \mathrm{H}), 4.18-4.13(\mathrm{~m}, 1 \mathrm{H}), 3.76(\mathrm{dt}, \mathrm{J}$ =12, $2.7 \mathrm{~Hz}, 1 \mathrm{H})$, 2.17-2.04 (m, $2 \mathrm{H}), 1.81-1.57$ (m, $4 \mathrm{H})$; LC-MS $299\left(\mathrm{MH}^{+}\right)$. Anal. $\left(\mathrm{C}_{18} \mathrm{H}_{14} \mathrm{ClF}_{3} \mathrm{~N}_{2} \mathrm{O}_{2}\right) \mathrm{C}, \mathrm{H}, \mathrm{N}$. The following alkyne derivatives 11 were prepared using the same procedure.

\section{4-Chloro-2-(tetrahydropyran-2-yl)-5-(4-chlorophenylethynyl)-2H-pyridazin-3-one} (11b). ${ }^{1} \mathrm{H}$ NMR $\left(300 \mathrm{MHz}, \mathrm{CDCl}_{3}\right) \delta 7.84$ (s, $\left.1 \mathrm{H}\right), 7.56-7.51$ (m, $\left.2 \mathrm{H}\right), 7.41-7.37$ (m, 2 H), 6.06 (dd, J = 11, 2.5 Hz, 1 H), 4.18-4.13 (m, $1 \mathrm{H}), 3.76$ (td, J = 12, $2.7 \mathrm{~Hz}, 1 \mathrm{H}), 2.20$ $2.00(\mathrm{~m}, 2 \mathrm{H}), 1.80-1.63(\mathrm{~m}, 3 \mathrm{H}), 1.61-1.58(\mathrm{~m}, 1 \mathrm{H})$. Anal. $\left(\mathrm{C}_{17} \mathrm{H}_{14} \mathrm{Cl}_{2} \mathrm{~N}_{2} \mathrm{O}_{2}\right) \mathrm{C}, \mathrm{H}, \mathrm{N}$.

\section{4-Chloro-2-(tetrahydropyran-2-yl)-5-(4-fluorophenylethynyl)-2H-pyridazin-3-one} (11c). ${ }^{1} \mathrm{H}$ NMR $\left(300 \mathrm{MHz}, \mathrm{CDCl}_{3}\right) \delta 7.84$ (s, $\left.1 \mathrm{H}\right), 7.63-7.57$ (m, $\left.2 \mathrm{H}\right), 7.15-7.07$ (m, 2 H), 6.06 (dd, J = 11, 2.0 Hz, 1 H), 4.18-4.13 (m, 1 H), 3.76 (td, J = 12, 2.7 Hz, 1 H), 2.22$2.12(\mathrm{~m}, 2 \mathrm{H}), 1.79-1.63(\mathrm{~m}, 3 \mathrm{H}), 1.62-1.56(\mathrm{~m}, 1 \mathrm{H})$. Anal. $\left(\mathrm{C}_{17} \mathrm{H}_{14} \mathrm{ClFN}_{2} \mathrm{O}_{2}\right) \mathrm{C}, \mathrm{H}, \mathrm{N}$.

\section{4-Chloro-2-(tetrahydropyran-2-yl)-5-(4-(methoxyphenyl)ethynyl)-2H-pyridazin-3- one (11d). ${ }^{1} \mathrm{H}$ NMR (300 MHz, $\left.\mathrm{CDCl}_{3}\right) \delta 7.83$ (s, $\left.1 \mathrm{H}\right)$, 7.57-7.52 (m, $\left.2 \mathrm{H}\right), 6.94-6.89$ (m, 2 H), $6.06(\mathrm{dd}, \mathrm{J}=11,2.1 \mathrm{~Hz}, 1 \mathrm{H}), 4.18-4.12$ (m, $1 \mathrm{H}), 3.85$ (s, $3 \mathrm{H}), 3.76$ (td, J =}


12, $2.4 \mathrm{~Hz}, 1 \mathrm{H}), 2.15-2.03(\mathrm{~m}, 2 \mathrm{H}), 1.78-1.56(\mathrm{~m}, 4 \mathrm{H})$. Anal. $\left(\mathrm{C}_{18} \mathrm{H}_{17} \mathrm{ClN}_{2} \mathrm{O}_{3} \cdot 1 / 3 \mathrm{H}_{2} \mathrm{O}\right)$ $\mathrm{C}, \mathrm{H}, \mathrm{N}$.

\section{4-Chloro-2-(tetrahydropyran-2-yl)-5-(4-ethylphenylethynyl)-2H-pyridazin-3-one} (11e). ${ }^{1} \mathrm{H}$ NMR (300 MHz, $\left.\mathrm{CDCl}_{3}\right) \delta 7.84$ (s, $\left.1 \mathrm{H}\right), 7.53$ (d, J = 8.4 Hz, $\left.2 \mathrm{H}\right), 7.24$ (d, J = $8.4 \mathrm{~Hz}, 2 \mathrm{H}), 6.06$ (dd, J = 11, $2.1 \mathrm{~Hz}, 1 \mathrm{H}), 4.18-4.13$ (m, 1 H), 3.76 (td, J = 12, $2.8 \mathrm{~Hz}, 1$ H), 2.69 (q, J = 7.6 Hz, 2 H), 2.17-2.03 (m, 3 H), 1.81-1.55 (m, 3 H), 1.25 (t, J = 7.6 Hz, $3 \mathrm{H})$; LC-MS 259 ( $\mathrm{MH}^{+}$-tetrahydropyran).

2-(4-Trifluoromethylphenyl)-6H-furo[2,3-d]pyridazin-7-one (12a). Alkyne 11a (383 $\mathrm{mg}, 1.0 \mathrm{mmol}$ ) was dissolved in dioxane $(9 \mathrm{~mL})$ and treated with potassium hydroxide (710 mg, $11 \mathrm{mmol})$ in water $(4.5 \mathrm{~mL})$. The mixture was heated to reflux for $3 \mathrm{~h}$, cooled, diluted with water $(60 \mathrm{~mL})$ and extracted three times with ethyl acetate. The combined extracts were dried $\left(\mathrm{MgSO}_{4}\right)$ and concentrated under vacuum. The residue was purified by flash chromatography (elution with $25 \%$ ethyl acetate in hexaneS) to afford $134 \mathrm{mg}$ (37\%) of the THP derivative of 12a as a white solid: ${ }^{1} \mathrm{H}$ NMR $\left(300 \mathrm{MHz}, \mathrm{CDCl}_{3}\right) \delta 8.27$ (s, $1 \mathrm{H}), 8.02(\mathrm{~d}, \mathrm{~J}=8.1 \mathrm{~Hz}, 2 \mathrm{H}), 7.74(\mathrm{~d}, \mathrm{~J}=8.4 \mathrm{~Hz}, 2 \mathrm{H}), 7.04(\mathrm{~s}, 1 \mathrm{H}), 6.26$ (dd, J = 11, $2.3 \mathrm{~Hz}, 1 \mathrm{H}), 4.20-4.15(\mathrm{~m}, 1 \mathrm{H}), 3.82(\mathrm{td}, \mathrm{J}=12,2.7 \mathrm{~Hz}, 1 \mathrm{H}), 2.31-2.18(\mathrm{~m}, 1 \mathrm{H})$, 2.10-2.04 (m, 1 H), 1.86-1.74 (m, 3 H), 1.64-1.60 (m, 1 H); LC-MS $365\left(\mathrm{MH}^{+}\right)$. Anal. $\left(\mathrm{C}_{18} \mathrm{H}_{15} \mathrm{~F}_{3} \mathrm{~N}_{2} \mathrm{O}_{3}\right) \mathrm{C}, \mathrm{H}, \mathrm{N}$. This material was suspended in methanol $(2 \mathrm{~mL})$ and $6 \mathrm{~N}$ hydrochloric acid $(4 \mathrm{~mL})$ and heated to reflux. After 90 minutes, the mixture was cooled to room temperature and filtered. The white precipitate was washed with water and triturated with ethanol ( $3 \mathrm{~mL})$ to afford a quantitative yield of 12a which was used without further purification.

\section{1-Methyl-2-(4-trifluoromethylphenyl)-1,6-dihydropyrrolo[2,3-d]pyridazin-7-one}

(13a). Alkyne 11a (383 mg, $1.0 \mathrm{mmol})$, ethanol (20 mL), and $40 \%$ aqueous methylamine $(6.1 \mathrm{~mL})$ were combined and heated at $80^{\circ} \mathrm{C}$ in a sealed tube for $2 \mathrm{~h}$. The mixture was cooled to room temperature and the resulting white needles were isolated by filtration. This material was dissolved in DMA $(3 \mathrm{~mL})$ and ethylene glycol $(0.3 \mathrm{~mL})$ and treated with sodium hexamethyldisilylamide $(90 \mathrm{mg}, 0.49 \mathrm{mmol})$. The mixture was heated in a 
sealed tube at $80^{\circ} \mathrm{C}$ for $18 \mathrm{~h}$ and cooled to room temperature. It was diluted with ethyl acetate $(15 \mathrm{~mL})$ and washed twice with water and twice with aqueous sodium chloride. The organic layer was dried $\left(\mathrm{MgSO}_{4}\right)$ and concentrated and the residue was purified by flash chromatography (elution with $25 \%$ ethyl acetate in hexanes to afford $52 \mathrm{mg}$ (14\%) the THP derivative of 13a as a white solid: ${ }^{1} \mathrm{H}$ NMR $\left(300 \mathrm{MHz}, \mathrm{CDCl}_{3}\right) \delta 8.20(\mathrm{~s}, 1 \mathrm{H})$, 7.76 (d, J = 8.4 Hz, 2 H), 7.59 (d, J = 8.4 Hz, 2 H), 6.48 (s, 1 H), 6.20 (dd, J = 11, $2.3 \mathrm{~Hz}$, $1 \mathrm{H}), 4.20-4.14$ (m, $1 \mathrm{H}), 4.15$ (s, $3 \mathrm{H}), 3.80$ (td, J = 12, $2.8 \mathrm{~Hz}, 1 \mathrm{H}), 2.40-2.28(\mathrm{~m}, 1 \mathrm{H})$, 2.15-2.08 (m, $1 \mathrm{H})$, 1.85-1.68 (m, $3 \mathrm{H}), 1.63-1.52(\mathrm{~m}, 1 \mathrm{H})$; LC-MS $378\left(\mathrm{MH}^{+}\right)$. Anal. $\left(\mathrm{C}_{19} \mathrm{H}_{18} \mathrm{~F}_{3} \mathrm{~N}_{3} \mathrm{O}_{2}\right) \mathrm{C}, \mathrm{H}, \mathrm{N}$. This material was deprotected and used without further purification as described for 12a.

\section{2-(4-Trifluoromethylphenyl)-6H-thieno[2,3-d]pyridazin-7-one (14a). Compound 11a} (466 mg, $1.22 \mathrm{mmol}$ ) was dissolved in DMF (6 mL), sodium sulfide nonahydrate (643 $\mathrm{mg}, 2.68 \mathrm{mmol}$ ) was added, and the mixture was heated at $70^{\circ} \mathrm{C}$ for $1 \mathrm{~h}$. The mixture was poured into water $(24 \mathrm{~mL})$ and extracted four times with DCM $(15 \mathrm{~mL})$. The combined extracts were dried $\left(\mathrm{MgSO}_{4}\right)$, concentrated under vacuum, and the residue was purified by flash chromatography (elution with $20 \%$ ethyl acetate in hexaneS) to afford $422 \mathrm{mg}$ (91\%) of the title compound as a yellow powder: ${ }^{1} \mathrm{H}$ NMR (300 MHz, $\left.\mathrm{CDCl}_{3}\right) \delta 8.30$ (s, $1 \mathrm{H}), 7.81(\mathrm{~d}, \mathrm{~J}=8.4 \mathrm{~Hz}, 2 \mathrm{H}), 7.72(\mathrm{~d}, \mathrm{~J}=8.1 \mathrm{~Hz}, 2 \mathrm{H}), 7.55$ (s, $1 \mathrm{H}), 6.21$ (dd, J = 11, $2.1 \mathrm{~Hz}, 1 \mathrm{H}), 4.20-4.15$ (m, $1 \mathrm{H}), 3.85-3.76$ (m, $1 \mathrm{H}), 2.33-2.22$ (m, $1 \mathrm{H}), 2.10-2.04$ (m, 1 $\mathrm{H})$, 1.85-1.74 (m, $3 \mathrm{H})$, 1.65-1.57 (m, $1 \mathrm{H})$; LC-MS $381\left(\mathrm{MH}^{+}\right)$. Anal. $\left(\mathrm{C}_{18} \mathrm{H}_{15} \mathrm{~F}_{3} \mathrm{~N}_{2} \mathrm{O}_{2} \mathrm{~S}\right)$ $\mathrm{C}, \mathrm{H}, \mathrm{N}, \mathrm{S}$. This material was deprotected and used without further purification as described for 12a. Compounds 14b-e were prepared using the same procedure and showed LCMS data consistent with their structures.

\section{2-(4-Trifluoromethylphenyl)-6H-selenolo[2,3-d]pyridazin-7-one (15a). Alkyne 11a} (390 mg, $1.0 \mathrm{mmol}$ ) and elemental selenium (177 mg, 2.24 gatom) were suspended in DMF ( $8 \mathrm{~mL})$ and nitrogen was bubbled through the mixture for 10 minutes. The mixture was cooled in an ice-bath and sodium borohydride ( $85 \mathrm{mg}, 2.2 \mathrm{mmol}$ ) was added in one portion. After $20 \mathrm{~min}$, the mixture was poured into water $(40 \mathrm{~mL})$ and extracted with DCM $(40 \mathrm{~mL})$. The DCM extract was dried $\left(\mathrm{MgSO}_{4}\right)$ and concentrated. The residue was 
purified by flash chromatography (elution with $25 \%$ ethyl acetate in hexanes) to afford $148 \mathrm{mg}$ (34\%) of the THP derivative of $\mathbf{1 5 a}$ as a yellow powder: ${ }^{1} \mathrm{H} \mathrm{NMR}(300 \mathrm{MHz}$, $\left.\mathrm{CDCl}_{3}\right) \delta 8.24(\mathrm{~m}, 1 \mathrm{H}), 7.76-7.69(\mathrm{~m}, 5 \mathrm{H}), 6.19(\mathrm{dd}, \mathrm{J}=11,2.0 \mathrm{~Hz}, 1 \mathrm{H}), 4.20-4.15(\mathrm{~m}$, $1 \mathrm{H}), 3.80(\mathrm{td}, \mathrm{J}=12,2.5 \mathrm{~Hz}, 1 \mathrm{H}), 2.36-2.22(\mathrm{~m}, 1 \mathrm{H}), 2.10-2.00(\mathrm{~m}, 1 \mathrm{H}), 1.81-1.74(\mathrm{~m}$, $3 \mathrm{H})$, 1.64-1.56 (m, $1 \mathrm{H})$; LC-MS $429\left(\mathrm{MH}^{+}\right)$. Anal. $\left(\mathrm{C}_{18} \mathrm{H}_{15} \mathrm{~F}_{3} \mathrm{~N}_{2} \mathrm{O}_{2} \mathrm{Se}\right) \mathrm{C}, \mathrm{H}, \mathrm{N}$. This material was deprotected and used without further purification as described for 12a.

\section{[(S)-1-(5-Bromopyridin-2-yl)pyrrolidin-3-yl]dimethylamine ((S)-17a).}

Toluenesulfonic acid monohydrate $(0.31 \mathrm{~g}, 1.6 \mathrm{mmol}),(3 S)-3-$ (dimethylamino)pyrrolidine ( $0.53 \mathrm{~g}, 4.66 \mathrm{mmol})$ and 2,5-dibromopyridine $(1.1 \mathrm{~g}, 4.6$ mmol) were combined and heated at $130^{\circ} \mathrm{C}$ for $20 \mathrm{~h}$. The mixture was cooled and the resulting material was purified by flash chromatography (elution with $2 \%$ methanol and $0.5 \%$ aqueous ammonia in DCM) to afford $0.30 \mathrm{~g}(24 \%)$ of $(\boldsymbol{S})$-17a as an off-white solid: ${ }^{1} \mathrm{H}$ NMR $\left(300 \mathrm{MHz}, \mathrm{CDCl}_{3}\right) \delta 8.15(\mathrm{~d}, \mathrm{~J}=2.7 \mathrm{~Hz}, 1 \mathrm{H}), 7.48(\mathrm{dd}, \mathrm{J}=9.0,2.4 \mathrm{~Hz}, 1 \mathrm{H})$, $6.25(\mathrm{~d}, \mathrm{~J}=9.0 \mathrm{~Hz}, 1 \mathrm{H}), 3.72(\mathrm{dd}, \mathrm{J}=9.9,7.2 \mathrm{~Hz}, 1 \mathrm{H}), 3.59(\mathrm{td}, \mathrm{J}=8.1,1.6 \mathrm{~Hz}, 1 \mathrm{H})$, $3.36(\mathrm{td}, \mathrm{J}=10,4.2 \mathrm{~Hz}, 1 \mathrm{H}), 3.21(\mathrm{dd}, \mathrm{J}=9.9,8.7 \mathrm{~Hz}, 1 \mathrm{H}), 2.85-2.75(\mathrm{~m}, 1 \mathrm{H}), 2.31(\mathrm{~s}$, $6 \mathrm{H}), 2.30-2.19$ (m, $1 \mathrm{H}), 1.96-1.85(\mathrm{~m}, 1 \mathrm{H})$; LC-MS $\left(\mathrm{MH}^{+}\right)$270. Anal. $\left(\mathrm{C}_{11} \mathrm{H}_{16} \mathrm{BrN}_{3}\right) \mathrm{C}$, $\mathrm{H}, \mathrm{N}$. (R)-17a was prepared using the same procedure and provided LC-MS and NMR spectra that were indistinguishable from those of its enantiomer. Using this procedure the remaining bromopyridines 17 were prepared.

[(S)-1-(5-Bromopyridin-2-yl)pyrrolidin-3-yl]methylamine ((S)-17b). ${ }^{1} \mathrm{H}$ NMR (300 $\left.\mathrm{MHz}, \mathrm{CDCl}_{3}\right) \delta 8.14(\mathrm{~d}, \mathrm{~J}=2.4 \mathrm{~Hz}, 1 \mathrm{H}), 7.47(\mathrm{dd}, \mathrm{J}=8.9,2.6 \mathrm{~Hz}, 1 \mathrm{H}), 6.25(\mathrm{~d}, \mathrm{~J}=8.7$ Hz, $1 \mathrm{H}), 3.62$ (dd, J = 10, 5.9 Hz, $1 \mathrm{H}), 3.58-3.50$ (m, $1 \mathrm{H}$ ), 3.45-3.34 (m, $2 \mathrm{H}), 3.25$ (dd, $\mathrm{J}=9.9,4.8 \mathrm{~Hz}, 1 \mathrm{H}), 2.48$ (s, $3 \mathrm{H}), 2.26-2.15$ (m, $1 \mathrm{H}), 1.93-1.82$ (m, $1 \mathrm{H})$; LC-MS $\left(\mathrm{MH}^{+}\right)$256. Anal. $\left(\mathrm{C}_{10} \mathrm{H}_{14} \mathrm{BrN}_{3}\right) \mathrm{C}, \mathrm{H}, \mathrm{N}$. (R)-17b was prepared using the same procedure and provided LC-MS and NMR spectra that were indistinguishable from those of its enantiomer.

[(S)-1-(5-Bromopyridin-2-yl)pyrrolidin-3-yl]ethylamine ((S)-17c). ${ }^{1} \mathrm{H}$ NMR (300 $\left.\mathrm{MHz}, \mathrm{CDCl}_{3}\right) \delta 8.15(\mathrm{~d}, \mathrm{~J}=1.8 \mathrm{~Hz}, 1 \mathrm{H}), 7.47(\mathrm{dd}, \mathrm{J}=8.9,2.6 \mathrm{~Hz}, 1 \mathrm{H}), 6.25(\mathrm{~d}, \mathrm{~J}=8.7$ 
Hz, $1 \mathrm{H}), 3.69$ (dd, J = 10, 6.2 Hz, 1 H), 3.60-3.36 (m, 3 H), 3.24 (dd, J = 10, $5.3 \mathrm{~Hz}, 1$ H), $2.74(\mathrm{q}, \mathrm{J}=7.2 \mathrm{~Hz}, 2 \mathrm{H}), 2.29-2.19(\mathrm{~m}, 1 \mathrm{H}), 1.98-1.85(\mathrm{~m}, 1 \mathrm{H}), 1.15(\mathrm{t}, \mathrm{J}=6.9 \mathrm{~Hz}$, $3 \mathrm{H})$; LC-MS $\left(\mathrm{MH}^{+}\right) 270$.

(S)-1'-(5-Bromopyridin-2-yl)-[1,3']bipyrrolidinyl ((S)-17d). ${ }^{1} \mathrm{H}$ NMR $(300 \mathrm{MHz}$, $\left.\mathrm{CDCl}_{3}\right) \delta 8.14(\mathrm{~d}, \mathrm{~J}=2.1 \mathrm{~Hz}, 1 \mathrm{H}), 7.47(\mathrm{dd}, \mathrm{J}=9.0,2.4 \mathrm{~Hz}, 1 \mathrm{H}), 6.24(\mathrm{~d}, \mathrm{~J}=9.3 \mathrm{~Hz}, 1$ H), $3.70(\mathrm{dd}, \mathrm{J}=10,6.9 \mathrm{~Hz}, 1 \mathrm{H}), 3.61-3.54(\mathrm{~m}, 1 \mathrm{H}), 3.45-3.33(\mathrm{~m}, 1 \mathrm{H}), 3.28(\mathrm{dd}, \mathrm{J}=$ $10,8.1 \mathrm{~Hz}, 1 \mathrm{H}), 2.92-2.82$ (m, $1 \mathrm{H}), 2.62-2.43$ (m, $4 \mathrm{H}), 2.27-2.18$ (m, $2 \mathrm{H}), 2.08-1.78$ (m, 4 H); LC-MS $\left(\mathrm{MH}^{+}\right) 296$.

4-[(R)-1-(5-Bromopyridin-2-yl)pyrrolidin-3-yl]morpholine ((R)-17e). ${ }^{1} \mathrm{H}$ NMR (300 $\left.\mathrm{MHz}, \mathrm{CDCl}_{3}\right) \delta 8.16(\mathrm{~d}, \mathrm{~J}=2.4 \mathrm{~Hz}, 1 \mathrm{H}), 7.51(\mathrm{dd}, \mathrm{J}=8.9,2.5 \mathrm{~Hz}, 1 \mathrm{H}), 6.26(\mathrm{~d}, \mathrm{~J}=9.0$ $\mathrm{Hz}, 1 \mathrm{H}$ ), 4.00-3.65 (m, 4 H), 3.63 (t, J = 10 Hz, 2 H), 3.39 (q, J = 9.0 Hz, 2 H), 2.80-2.40 (m, $5 \mathrm{H}), 2.28-2.10$ (m, $2 \mathrm{H})$; LC-MS $\left(\mathrm{MH}^{+}\right) 312$.

\section{6-(4-Chlorophenyl)-2-[6-((R)-dimethylaminopyrrolidin-1-yl)pyridine-3-yl]-2H-} phthalazin-7-one hydrochloride $((\boldsymbol{R})-18 a)$ 6-(4-Chloro-phenyl)-2H-phthalazin-1-one (13.1 g, $51.2 \mathrm{mmol})$, [(R)-1-(5-bromo-pyridin-2-ylpyrrolidin-3-yl]dimethylamine (13.1 g, $48.6 \mathrm{mmol})$, cesium carbonate $(33.3 \mathrm{~g}, 102 \mathrm{mmol})$, copper iodide (9.77 g, $51.4 \mathrm{mmol})$ and ( \pm -trans-1,2-cyclohexanediamine $(10.2 \mathrm{~g}, 89.2 \mathrm{mmol})$ were combined with nitrogen purged 1,4-dioxane $(500 \mathrm{~mL})$ and heated to $110^{\circ} \mathrm{C}$ with stirring in a sealed pressure vessel. After $9 \mathrm{~h}$, the mixture was cooled to room temperature, combined with dichloromethane $(400 \mathrm{~mL})$ and ammonium hydroxide $(200 \mathrm{~mL})$, and stirred vigorously for $10 \mathrm{~min}$. The aqueous layer was separated and extracted three times with dichloromethane. The combined organic extracts were concentrated in vacuo and the residue was purified by silica gel chromatography (elution with $4 \%$ methanol and $0.5 \%$ aqueous ammonia in DCM) to afford the title compound as a yellow solid (8.37 g, 39\%). A portion of this material was converted to the hydrochloride salt as described in the general experimental section. ${ }^{1} \mathrm{H}$ NMR (300 MHz, DMSO-d $\left.{ }_{6}\right) \delta 11.35$ (br s, $\left.1 \mathrm{H}\right), 8.62$ (s, $1 \mathrm{H}), 8.39-8.35$ (m, $3 \mathrm{H}), 8.23$ (dd, J = 8.1, $1.8 \mathrm{~Hz}, 1 \mathrm{H}), 8.04$ (d, J = 8.7 Hz, $1 \mathrm{H})$, 7.91-7.82 (m, 2 H), 7.65-7.62 (m, 2 H), 6.92 (d, J = 9.0 Hz, 1 H), 4.05-3.96 (m, 2 H), 
3.90-3.75 (m, 2 H), 3.60-3.49 (m, 1 H), 2.84-2.80 (m, 6 H), 2.48-2.39 (m, 2 H); LC-MS $\left(\mathrm{MH}^{+}\right)$446. Anal. $\left(\mathrm{C}_{25} \mathrm{H}_{24} \mathrm{ClN}_{5} \mathrm{O} \cdot 2 \mathrm{HCl} \cdot 2 \mathrm{H}_{2} \mathrm{O} \cdot \mathrm{C}_{2} \mathrm{H}_{6} \mathrm{O}\right) \mathrm{C}, \mathrm{H}, \mathrm{N}$. $(S)$-18a was prepared using the same procedure and provided LC-MS and NMR spectra that were indistinguishable from those of its enantiomer. Using this procedure and the starting materials $\mathbf{8}, \mathbf{1 2 - 1 5}$, and $\mathbf{1 7}$, the following compounds were prepared.

6-(4-Chlorophenyl)-2-[6-((S)-methylaminopyrrolidin-1-yl)pyridine-3-yl]-2Hphthalazin-7-one hydrochloride ((S)-18b). ${ }^{1} \mathrm{H}$ NMR $\left(300 \mathrm{MHz}, \mathrm{CDCl}_{3}\right) \delta 8.38$ (d, J = $2.7 \mathrm{~Hz}, 1 \mathrm{H}), 8.32(\mathrm{~d}, \mathrm{~J}=8.4 \mathrm{~Hz}, 1 \mathrm{H}), 8.28(\mathrm{~s}, 1 \mathrm{H}), 8.23(\mathrm{dd}, \mathrm{J}=9.6,2.1 \mathrm{~Hz}, 1 \mathrm{H}), 7.90$ $(\mathrm{dd}, \mathrm{J}=8.4,1.8 \mathrm{~Hz}, 1 \mathrm{H}), 7.84(\mathrm{~s}, 1 \mathrm{H}), 7.49(\mathrm{~d}, \mathrm{~J}=8.7,2 \mathrm{H}), 7.33(\mathrm{~d}, \mathrm{~J}=8.7,2 \mathrm{H}), 6.94$ (d, J = 9.6 Hz, 1 H), 3.93-3.81 (m, 4 H), 3.63-3.55 (m, 1 H), 2.62 (s, 3 H), 2.48-2.24 (m, 2 $\mathrm{H})$; LC-MS $\left(\mathrm{MH}^{+}\right)$432. Anal. $\left(\mathrm{C}_{24} \mathrm{H}_{22} \mathrm{ClN}_{5} \mathrm{O} \cdot \mathrm{HCl} \cdot 3 \mathrm{H}_{2} \mathrm{O}\right)$ calcd: $\mathrm{C}, 55.18 ; \mathrm{H}, 5.59 ; \mathrm{N}$, 13.40. Found: C, 54.44; H, 5.60; $\mathrm{N}, 13.74$. $(\boldsymbol{R})-\mathbf{1 8 b}$ was prepared using the same procedure and provided LC-MS and NMR spectra that were indistinguishable from those of its enantiomer.

6-(4-Chlorophenyl)-2-[6-((S)-ethylaminopyrrolidin-1-yl)pyridine-3-yl]-2Hphthalazin-7-one hydrotrifluoroacetate $\left((\boldsymbol{S})\right.$-18c). ${ }^{1} \mathrm{H} \mathrm{NMR}\left(300 \mathrm{MHz}, \mathrm{CDCl}_{3}\right) \delta$ 10.00 (br s, 1 H), 8.58 (s, 1 H), 8.43 (d, J = 8.4 Hz, 1 H), 8.31 (s, 1 H), 8.18 (d, J = 9.6 $\mathrm{Hz}, 1 \mathrm{H}$ ), 7.89 (dd, J = 8.4, $1.8 \mathrm{~Hz}, 1 \mathrm{H}$ ), 7.85 (d, J = 1.2 Hz, $1 \mathrm{H}$ ), 7.57 (d, J = 9.0 Hz, 2 H), 7.47 (d, J = 8.7 Hz, 2 H), 6.77 (d, J = 9.6 Hz, 1 H), 4.66-4.25 (m, 2 H), 4.22-4.10 (m, $1 \mathrm{H}), 4.10-3.92$ (m, $3 \mathrm{H}), 3.74-3.64$ (m, $1 \mathrm{H}), 3.20-3.10$ (m, $2 \mathrm{H}), 2.62-2.42(\mathrm{~m}, 2 \mathrm{H})$, 1.40-1.30 (m, $3 \mathrm{H})$; LC-MS ( $\left.\mathrm{t}_{\mathrm{R}}, \mathrm{MH}^{+}\right) 4.922 \mathrm{~min}, 446$ (method 1). HRMS calcd for $\mathrm{C}_{25} \mathrm{H}_{25} \mathrm{ClN}_{5} \mathrm{O}\left[\mathrm{MH}^{+}\right]$446.1742, found 446.1746 .

2-((S)-6-[1,3']Bipyrrolidinyl-1'-yl-pyridin-3-yl)-6-(4-chlorophenyl)-2H-phthalazin-7one hydrochloride $((\boldsymbol{S})-\mathbf{1 8 d}) .{ }^{1} \mathrm{H} \mathrm{NMR}\left(300 \mathrm{MHz}, 4: 1 \mathrm{CDCl}_{3} / \mathrm{CD}_{3} \mathrm{OD}\right) \delta 8.46(\mathrm{~d}, \mathrm{~J}=$ $8.4 \mathrm{~Hz}, 1 \mathrm{H}), 8.40-8.37$ (m, $1 \mathrm{H}), 8.03(\mathrm{dd}, \mathrm{J}=8.4,1.8 \mathrm{~Hz}, 1 \mathrm{H}), 7.98(\mathrm{~d}, \mathrm{~J}=1.8 \mathrm{~Hz}, 1 \mathrm{H})$, $7.81(\mathrm{dd}, \mathrm{J}=9.3,2.7 \mathrm{~Hz}, 1 \mathrm{H}), 7.72-7.58$ (m, $2 \mathrm{H}), 7.50-7.44$ (m, 2 H), 6.80-6.66 (m, 1 H), $6.58(\mathrm{~d}, \mathrm{~J}=8.7 \mathrm{~Hz}, 1 \mathrm{H}), 4.04-3.93(\mathrm{~m}, 2 \mathrm{H}), 3.87-3.76(\mathrm{~m}, 2 \mathrm{H}), 3.76-3.59(\mathrm{~m}, 1 \mathrm{H})$, 3.59-3.49 (m, 2 H), 3.29-3.17 (m, 2 H), 2.56-2.52 (m, 2 H), 2.17-2.13 (m, 3 H), 0.95-0.81 
(m, $1 \mathrm{H}$ ); LC-MS $\left(\mathrm{t}_{\mathrm{R}}, \mathrm{MH}^{+}\right) 4.820$ min, 472 (method 1). HRMS calcd for $\mathrm{C}_{27} \mathrm{H}_{26} \mathrm{ClN}_{5} \mathrm{O}$ $\left[\mathrm{MH}^{+}\right]$472.1899, found 472.1889.

6-(4-Chlorophenyl)-2-[6-((R)-3-morpholin-4-yl-pyrrolidin-1-yl)pyridine-3-yl]-2Hphthalazin-7-one hydrotrifluoroacetate ((R)-18e). ${ }^{1} \mathrm{H}$ NMR $\left(300 \mathrm{MHz}, \mathrm{DMSO}_{6} \mathrm{~d}_{6}\right)$ $\delta 10.10($ br s, $1 \mathrm{H}), 8.58(\mathrm{~s}, 1 \mathrm{H}) ; 8.39-8.31(\mathrm{~m}, 3 \mathrm{H}), 8.22$ (dd, J = 8.4, $1.8 \mathrm{~Hz}, 1 \mathrm{H}) ; 7.89$ $(\mathrm{d}, \mathrm{J}=8.7 \mathrm{~Hz}, 2 \mathrm{H}) ; 7.80(\mathrm{dd}, \mathrm{J}=8.4,1.8 \mathrm{~Hz}, 1 \mathrm{H}), 7.63(\mathrm{~d}, \mathrm{~J}=8.7 \mathrm{~Hz}, 2 \mathrm{H}) ; 6.66(\mathrm{~d}, \mathrm{~J}=$ $9.1 \mathrm{~Hz}, 1 \mathrm{H}$ ): 4.14-3.13 (m, $14 \mathrm{H}$ ): 2.25 (br s, $1 \mathrm{H}$ ); LC-MS (t $\left.\mathrm{t}_{\mathrm{R}}, \mathrm{MH}^{+}\right) 5.150$ min, 488 (method 1). HRMS calcd for $\mathrm{C}_{27} \mathrm{H}_{26} \mathrm{ClN}_{5} \mathrm{O}_{2}\left[\mathrm{MH}^{+}\right]$488.1848, found 488.1843 .

6-(4-Trifluoromethylphenyl)-2-[6-((S)-methylaminopyrrolidin-1-yl)pyridine-3-yl]2H-phthalazin-7-one hydromethanesulfonate ((S)-18f). ${ }^{1} \mathrm{H}$ NMR (300 MHz, DMSO$\left.d_{6}\right): \delta 8.79$ (br s, $\left.2 \mathrm{H}\right), 8.60$ (s, $\left.1 \mathrm{H}\right), 8.44-8.30(\mathrm{~m}, 3 \mathrm{H}), 8.25$ (dd, J = 8.7, $1.8 \mathrm{~Hz}, 1 \mathrm{H}$ ), 8.07 (d, J = 7.9 Hz, 2 H), 7.91 (d, J = 7.9 Hz, 2 H), 7.79 (dd, J = 9.0, 2.6 Hz, 1 H), 6.65 (d, J = 8.7 Hz, $1 \mathrm{H}), 3.91(\mathrm{~m}, 1 \mathrm{H}), 3.78(\mathrm{~m}, 1 \mathrm{H}), 3.69-3.58(\mathrm{~m}, 2 \mathrm{H}), 3.51(\mathrm{~m}, 1 \mathrm{H}), 2.67$ (s, $3 \mathrm{H}), 2.39$ (m, $1 \mathrm{H}), 2.34$ (s, $3 \mathrm{H}), 2.21$ (m, $1 \mathrm{H})$; LC-MS (MH+) 466. Anal. $\left(\mathrm{C}_{25} \mathrm{H}_{22} \mathrm{~F}_{3} \mathrm{~N}_{5} \mathrm{O} \cdot \mathrm{CH}_{3} \mathrm{SO}_{3} \mathrm{H} \cdot 1 / 2 \mathrm{H}_{2} \mathrm{O}\right) \mathrm{C}, \mathrm{H}, \mathrm{N}, \mathrm{S}$. (R)-18f was prepared using the same procedure and provided LC-MS and NMR spectra that were indistinguishable from those of its enantiomer.

6-(4-Methoxy-2-methylphenyl)-2-[6-((S)-methylaminopyrrolidin-1-yl)pyridine-3-yl]2H-phthalazin-7-one hydrochloride ((S)-18g). ${ }^{1} \mathrm{H}$ NMR (300 MHz, $\left.\mathrm{CDCl}_{3}\right) \delta 8.34(\mathrm{~d}$, $\mathrm{J}=5.4 \mathrm{~Hz}, 1 \mathrm{H}), 8.27(\mathrm{~d}, \mathrm{~J}=2.4 \mathrm{~Hz}, 1 \mathrm{H}), 8.24(\mathrm{~s}, 1 \mathrm{H}), 7.72-7.66(\mathrm{~m}, 2 \mathrm{H}), 7.60-7.59$ (m, $1 \mathrm{H}), 7.10(\mathrm{~d}, \mathrm{~J}=5.4 \mathrm{~Hz}, 1 \mathrm{H}), 6.76-6.72(\mathrm{~m}, 2 \mathrm{H}), 6.47(\mathrm{~d}, \mathrm{~J}=6.3 \mathrm{~Hz}, 1 \mathrm{H}), 3.75(\mathrm{~s}$, 3 H), 3.66-3.42 (m, 5 H), 2.63 (s, 3 H), 2.46-2.36 (m, 1 H), 2.30-2.20 (m, 1 H), 2.18 (s, 3 $\mathrm{H})$; LC-MS $\left(\mathrm{t}_{\mathrm{R}}, \mathrm{MH}^{+}\right) 1.788$ min, 442 (method 2). HRMS calcd for $\mathrm{C}_{26} \mathrm{H}_{27} \mathrm{~N}_{5} \mathrm{O}_{2}\left[\mathrm{MH}^{+}\right]$ 442.2237, found 442.2245.

6-[6-((S)-Methylaminopyrrolidin-1-yl)pyridine-3-yl]-2-(4-trifluoromethylphenyl)6H-furo[2,3-d]pyridazin-7-one ((S)-19a). ${ }^{1} \mathrm{H}$ NMR (300 MHz, 4:1 $\left.\mathrm{CDCl}_{3} / \mathrm{CD}_{3} \mathrm{OD}\right)$ $\delta 8.33(\mathrm{~d}, \mathrm{~J}=2.7 \mathrm{~Hz}, 1 \mathrm{H}), 8.28(\mathrm{~s}, 1 \mathrm{H}), 7.97(\mathrm{~d}, \mathrm{~J}=5.4 \mathrm{~Hz}, 2 \mathrm{H}), 7.76(\mathrm{dd}, \mathrm{J}=9.3,2.7$ 
Hz, $1 \mathrm{H}), 7.68(\mathrm{~d}, \mathrm{j}=5.4 \mathrm{~Hz}, 2 \mathrm{H}), 7.10(\mathrm{~s}, 1 \mathrm{H}), 6.50(\mathrm{~d}, \mathrm{~J}=9.0 \mathrm{~Hz}, 1 \mathrm{H}), 3.95-3.76(\mathrm{~m}$, $3 \mathrm{H}), 3.62-3.43$ (m, $2 \mathrm{H}), 2.82$ (s, $6 \mathrm{H}), 2.50-2.96(\mathrm{~m}, 2 \mathrm{H})$; LC-MS ( $\left.\mathrm{t}_{\mathrm{R}}, \mathrm{MH}^{+}\right) 4.851 \mathrm{~min}$, 470 (method 1). HRMS calcd for $\mathrm{C}_{24} \mathrm{H}_{22} \mathrm{~F}_{3} \mathrm{~N}_{5} \mathrm{O}_{2}\left[\mathrm{MH}^{+}\right] 470.1798$, found 470.1797 .

1-Methyl-6-[6-((S)-methylaminopyrrolidin-1-yl)pyridine-3-yl]-2-(4trifluoromethylphenyl)-1,6-dihydropyrrolo[2,3-d]pyridazin-7-one ((S)-20a). ${ }^{1} \mathrm{H}$ NMR (300 MHz, 4:1 CDCl $\left./ \mathrm{CD}_{3} \mathrm{OD}\right) \delta 8.30(\mathrm{~d}, \mathrm{~J}=2.4 \mathrm{~Hz}, 1 \mathrm{H}), 8.18(\mathrm{~s}, 1 \mathrm{H}), 7.83$ (dd, $\mathrm{J}=9.0,2.7 \mathrm{~Hz}, 1 \mathrm{H}), 7.70(\mathrm{~d}, \mathrm{~J}=8.1 \mathrm{~Hz} 2 \mathrm{H}), 7.54(\mathrm{~d}, \mathrm{~J}=8.4 \mathrm{~Hz}, 2 \mathrm{H}), 6.58$ (d, J = 9.0 Hz, 1 H), 6.51 (s, 1 H), 4.05 (s, 3 H), 3.98-3.79 (m, 2 H), 3.64-3.47 (m, 3 H), 2.83 (s, 6 $\mathrm{H}), 2.50-2.37(\mathrm{~m}, 2 \mathrm{H})$; LC-MS ( $\left.\mathrm{t}_{\mathrm{R}}, \mathrm{MH}^{+}\right) 5.041 \mathrm{~min}, 483$ (method 1). HRMS calcd for $\mathrm{C}_{25} \mathrm{H}_{25} \mathrm{~F}_{3} \mathrm{~N}_{6} \mathrm{O}_{2}\left[\mathrm{MH}^{+}\right]$483.2115, found 483.2129.

6-[6-((S)-Dimethylaminopyrrolidin-1-yl)pyridine-3-yl]-2-(4-trifluoromethylphenyl)6H-thieno[2,3-d]pyridazin-7-one ((S)-21a). ${ }^{1} \mathrm{H}$ NMR (300 MHz, DMSO- $\left.d_{6}\right): \delta 10.00$ (br s, $1 \mathrm{H}), 8.60(\mathrm{~s}, 1 \mathrm{H}), 8.33$ (d, J = 2.6 Hz, $1 \mathrm{H}), 8.17(\mathrm{~s}, 1 \mathrm{H}), 8.10(\mathrm{~d}, \mathrm{~J}=8.2 \mathrm{~Hz}, 2 \mathrm{H})$, $7.89(\mathrm{~d}, \mathrm{~J}=8.2 \mathrm{~Hz}, 2 \mathrm{H}), 7.82(\mathrm{dd}, \mathrm{J}=9.0,2.6 \mathrm{~Hz}, 1 \mathrm{H}), 6.68(\mathrm{~d}, \mathrm{~J}=9.0 \mathrm{~Hz}, 1 \mathrm{H}), 4.05-$ 3.89 (m, 2 H), 3.73-3.58 (m, 2 H), 3.45 (m, 1 H), 2.87 (br s, 6 H), 2.45 (m, 1 H), 2.24 (m, $1 \mathrm{H})$; LC-MS $\left(\mathrm{MH}^{+}\right)$486. Anal. $\left(\mathrm{C}_{24} \mathrm{H}_{22} \mathrm{~F}_{3} \mathrm{~N}_{5} \mathrm{OS} \cdot 11 / 4 \mathrm{CF}_{3} \mathrm{CO}_{2} \mathrm{H}\right) \mathrm{C}, \mathrm{H}, \mathrm{N}, \mathrm{S}$.

6-[6-((R)-Methylaminopyrrolidin-1-yl)pyridine-3-yl]-2-(4-chlorophenyl)-6Hthieno[2,3-d]pyridazin-7-one ((R)-21b). LC-MS $\left(\mathrm{t}_{\mathrm{R}}, \mathrm{MH}^{+}\right) 4.630$ min, 438 (method 1).

6-[6-((S)-Methylaminopyrrolidin-1-yl)pyridine-3-yl]-2-(4-fluorophenyl)-6Hthieno[2,3-d]pyridazin-7-one hydrochloride ((S)-21c). ${ }^{1} \mathrm{H}$ NMR $\left(300 \mathrm{MHz}, \mathrm{CD}_{3} \mathrm{OD}\right)$ $\delta$ 857-8.56 (m, 2 H), $8.44(\mathrm{dd}, \mathrm{J}=9.9,2.4 \mathrm{~Hz}, 1 \mathrm{H}), 7.92-7.87$ (m, 2 H), $7.84(\mathrm{~s}, 1 \mathrm{H})$, 7.32-7.25 (m, 3 H), 4.16-4.08 (m, 2 H), 3.99-3.90 (m, 2 H), 3.88-3.79 (m, 1 H), 2.85 (s, 3 $\mathrm{H})$, 2.74-2.62 (m, $1 \mathrm{H}), 2.49-2.42$ (m, $1 \mathrm{H})$; LC-MS $\left(\mathrm{MH}^{+}\right)$427. Anal. $\left(\mathrm{C}_{22} \mathrm{H}_{20} \mathrm{FN}_{5} \mathrm{OS} \cdot 2 \mathrm{HCl}\right) \mathrm{C}, \mathrm{H}, \mathrm{N}, \mathrm{S}$. (R)-21c was prepared using the same procedure and provided LC-MS and NMR spectra that were indistinguishable from those of its enantiomer. 
6-[6-(R)-Methylaminopyrrolidin-1-yl)pyridine-3-yl]-2-(4-trifluoromethylphenyl)6H-thieno[2,3-d]pyridazin-7-one hydrochloride ((R)-21d). ${ }^{1} \mathrm{H}$ NMR (300 MHz, 4:1 $\left.\mathrm{CDCl}_{3} / \mathrm{CD}_{3} \mathrm{OD}\right) \delta 8.48(\mathrm{~d}, \mathrm{~J}=2.7 \mathrm{~Hz}, 1 \mathrm{H}), 8.37(\mathrm{dd}, \mathrm{J}=10.2,2.1 \mathrm{~Hz}, 1 \mathrm{H}), 8.36(\mathrm{~s}, 1 \mathrm{H})$, $7.78(\mathrm{~d}, \mathrm{~J}=8.4 \mathrm{~Hz}, 2 \mathrm{H}), 7.68(\mathrm{~d}, \mathrm{~J}=8.4 \mathrm{~Hz}, 2 \mathrm{H}), 7.68(\mathrm{~d}, \mathrm{~J}=8.4 \mathrm{~Hz}, 2 \mathrm{H}), 7.62(\mathrm{~s}, 1$ H), $7.06(\mathrm{~d}, \mathrm{~J}=9.6 \mathrm{~Hz}, 1 \mathrm{H}), 4.10-3.98(\mathrm{~m}, 4 \mathrm{H}), 3.75-3.67(\mathrm{~m}, 1 \mathrm{H}), 2.72$ (s, $3 \mathrm{H}), 2.60$ 2.48 (m, $2 \mathrm{H})$; LC-MS $\left(\mathrm{MH}^{+}\right)$472. Anal. $\left(\mathrm{C}_{20} \mathrm{H}_{20} \mathrm{~F}_{3} \mathrm{~N}_{5} \mathrm{OS} \cdot 2 \mathrm{HCl} \cdot 1 / 2 \mathrm{H}_{2} \mathrm{O}\right) \mathrm{C}, \mathrm{H}, \mathrm{N}, \mathrm{S}$. (S)21d was prepared using the same procedure and provided LC-MS and NMR spectra that were indistinguishable from those of its enantiomer.

6-[6-((R)-Methylaminopyrrolidin-1-yl)pyridine-3-yl]-2-(4-methoxyphenyl)-6Hthieno[2,3-d]pyridazin-7-one $((\boldsymbol{R})-21 \mathrm{e})$. LC-MS $\left(\mathrm{t}_{\mathrm{R}}, \mathrm{MH}^{+}\right) 4.100 \mathrm{~min}, 434(\operatorname{method} 1)$.

6-[6-((R)-Methylaminopyrrolidin-1-yl)pyridine-3-yl]-2-(4-ethylphenyl)-6Hthieno[2,3-d]pyridazin-7-one $(\boldsymbol{R})-21 f)$. LC-MS ( $\left.\mathrm{t}_{\mathrm{R}}, \mathrm{MH}^{+}\right) 1.920 \mathrm{~min}, 472$ (method 2).

6-[6-((S)-Methylaminopyrrolidin-1-yl)pyridine-3-yl]-2-(4-trifluoromethylphenyl)6H-selenolo[2,3-d]pyridazin-7-one hydrochloride ((S)-22a). ${ }^{1} \mathrm{H}$ NMR (300 MHz, 4:1 $\left.\mathrm{CDCl}_{3} / \mathrm{CD}_{3} \mathrm{OD}\right) \delta 8.38(\mathrm{~d}, \mathrm{~J}=2.1 \mathrm{~Hz}, 1 \mathrm{H}), 8.25(\mathrm{~s}, 1 \mathrm{H}), 7.85(\mathrm{dd}, \mathrm{J}=9.3 \mathrm{~Hz}, 2.7 \mathrm{~Hz}, 1$ H), $7.76(\mathrm{~s}, 1 \mathrm{H}), 7.71(\mathrm{~d}, \mathrm{~J}=5.4 \mathrm{~Hz}, 2 \mathrm{H}), 7.64(\mathrm{~d}, \mathrm{~J}=5.4 \mathrm{~Hz}, 2 \mathrm{H}), 6.55(\mathrm{~d}, \mathrm{~J}=6.3 \mathrm{~Hz}$, $1 \mathrm{H}), 3.95-3.79$ (m, $4 \mathrm{H}), 3.54-3.46$ (m, $1 \mathrm{H}), 2.83$ (s, $6 \mathrm{H}), 2.47-2.42$ (m, $2 \mathrm{H})$; LC-MS $\left(\mathrm{t}_{\mathrm{R}}, \mathrm{MH}^{+}\right) 2.196$ min, 534 (method 2). HRMS calcd for $\mathrm{C}_{24} \mathrm{H}_{22} \mathrm{~F}_{3} \mathrm{~N}_{5} \mathrm{OSe}\left[\mathrm{MH}^{+}\right]$534.1014, found 534.0995. 
Table of Elemental Analysis Data

\begin{tabular}{|c|c|c|c|c|c|c|c|c|c|}
\hline Cmpd & Formula & $\begin{array}{c}\% \mathrm{C} \\
\text { Calcd }\end{array}$ & $\begin{array}{c}\% \mathrm{C} \\
\text { Found }\end{array}$ & $\begin{array}{c}\% \mathrm{H} \\
\text { Calcd }\end{array}$ & $\begin{array}{c}\% \mathrm{H} \\
\text { Found }\end{array}$ & $\begin{array}{c}\% \mathrm{~N} \\
\text { Calcd }\end{array}$ & $\begin{array}{c}\% \mathrm{~N} \\
\text { Found }\end{array}$ & $\begin{array}{c}\% \mathrm{~S} \\
\text { Calcd }\end{array}$ & $\begin{array}{c}\% S \\
\text { Found }\end{array}$ \\
\hline $11 a$ & $\mathrm{C}_{18} \mathrm{H}_{14} \mathrm{ClF}_{3} \mathrm{~N}_{2} \mathrm{O}_{2}$ & 56.48 & 56.16 & 3.69 & 4.07 & 7.32 & 7.49 & - & - \\
\hline 11b & $\mathrm{C}_{17} \mathrm{H}_{14} \mathrm{Cl}_{2} \mathrm{~N}_{2} \mathrm{O}_{2}$ & 58.47 & 58.86 & 4.04 & 3.67 & 8.02 & 7.65 & - & - \\
\hline 11c & $\mathrm{C}_{17} \mathrm{H}_{14} \mathrm{ClFN}_{2} \mathrm{O}_{2}$ & 61.36 & 60.98 & 4.24 & 4.04 & 8.42 & 8.38 & - & - \\
\hline 11d & $\mathrm{C}_{18} \mathrm{H}_{17} \mathrm{ClN}_{2} \mathrm{O}_{3} \cdot 1 / 3 \mathrm{H}_{2} \mathrm{O}$ & 61.63 & 61.77 & 5.08 & 5.47 & 7.99 & 8.14 & - & - \\
\hline 12a-Thp ${ }^{a}$ & $\mathrm{C}_{18} \mathrm{H}_{15} \mathrm{~F}_{3} \mathrm{~N}_{2} \mathrm{O}_{2}$ & 59.34 & 59.27 & 4.15 & 4.49 & 7.69 & 7.80 & - & - \\
\hline 13a-Thp ${ }^{b}$ & $\mathrm{C}_{19} \mathrm{H}_{18} \mathrm{~F}_{3} \mathrm{~N}_{3} \mathrm{O}_{2}$ & 60.47 & 60.29 & 4.81 & 5.28 & 11.14 & 11.15 & - & - \\
\hline 14a-Thp & $\mathrm{C}_{18} \mathrm{H}_{15} \mathrm{~F}_{3} \mathrm{~N}_{2} \mathrm{O}_{2} \mathrm{~S}$ & 56.84 & 56.71 & 3.97 & 4.27 & 7.36 & 7.50 & 8.43 & 8.45 \\
\hline 15a-Thp & $\mathrm{C}_{18} \mathrm{H}_{15} \mathrm{~F}_{3} \mathrm{~N}_{2} \mathrm{O}_{2} \mathrm{Se}$ & 50.60 & 50.62 & 3.54 & 3.60 & 6.56 & 6.79 & - & - \\
\hline$(S)-17 a$ & $\mathrm{C}_{11} \mathrm{H}_{16} \mathrm{BrN}_{3}$ & 48.90 & 48.52 & 5.97 & 6.34 & 15.55 & 15.52 & - & - \\
\hline$(S)-17 b$ & $\mathrm{C}_{10} \mathrm{H}_{14} \mathrm{BrN}_{3}$ & 46.89 & 46.51 & 5.51 & 5.69 & 16.40 & 16.33 & - & - \\
\hline$(R)-18 a$ & $\mathrm{C}_{25} \mathrm{H}_{24} \mathrm{ClN}_{5} \mathrm{O} \cdot 2 \mathrm{HCl} \cdot 2 \mathrm{H}_{2} \mathrm{O} \cdot \mathrm{C}_{2} \mathrm{H}_{6} \mathrm{O}$ & 53.96 & 53.65 & 6.04 & 6.17 & 11.65 & 11.88 & - & - \\
\hline$(S)-18 b$ & $\mathrm{C}_{24} \mathrm{H}_{22} \mathrm{ClN}_{5} \mathrm{O} \cdot \mathrm{HCl} \cdot 3 \mathrm{H}_{2} \mathrm{O}$ & 55.18 & 54.44 & 5.59 & 5.60 & 13.40 & 13.74 & - & - \\
\hline$(S)-18 f$ & $\mathrm{C}_{25} \mathrm{H}_{22} \mathrm{~F}_{3} \mathrm{~N}_{5} \mathrm{O} \cdot \mathrm{CH}_{3} \mathrm{SO}_{3} \mathrm{H} \cdot 1 / 2 \mathrm{H}_{2} \mathrm{O}$ & 54.73 & 54.78 & 4.77 & 5.14 & 12.27 & 12.23 & 5.62 & 6.10 \\
\hline$(S)-21 a$ & $\mathrm{C}_{24} \mathrm{H}_{22} \mathrm{~F}_{3} \mathrm{~N}_{5} \mathrm{OS} \cdot 11 / 4 \mathrm{CF}_{3} \mathrm{CO}_{2} \mathrm{H}$ & 44.34 & 44.26 & 3.12 & 2.93 & 8.76 & 8.87 & 4.01 & 4.05 \\
\hline$(S)-21 c$ & $\mathrm{C}_{22} \mathrm{H}_{20} \mathrm{FN}_{5} \mathrm{OS} \cdot 2 \mathrm{HCl}$ & 55.34 & 53.21 & 4.48 & 4.22 & 14.16 & 14.31 & 6.48 & 6.26 \\
\hline$(R)-21 d$ & $\mathrm{C}_{23} \mathrm{H}_{20} \mathrm{~F}_{3} \mathrm{~N}_{5} \mathrm{OS} \cdot 2 \mathrm{HCl} \cdot 0.5 \mathrm{H}_{2} \mathrm{O}$ & 49.92 & 49.99 & 4.19 & 4.11 & 12.65 & 12.48 & 5.79 & 5.50 \\
\hline
\end{tabular}

${ }^{\mathrm{a}}$ Tetrahydropyranyl derivative of 12a. ${ }^{\mathrm{b}}$ Tetrahydropyranyl derivative of 13a. ${ }^{\mathrm{c}}$ Tetrahydropyranyl derivative of 14a. ${ }^{\mathrm{d}}$ Tetrahydropyranyl derivative of $\mathbf{1 5 a}$.

Biology. In vitro Binding. Competition radioligand binding experiments were performed on membrane preparations from chimeric rat/human MCH1R stably expressed in HEK293 cells. Cell membrane fractions were prepared as previously described and resuspended in assay buffer (50 mM HEPES, $10 \mathrm{mM} \mathrm{MgCl}_{2}, 2 \mathrm{mM}$ EDTA, $\left.\mathrm{pH} 7.0\right){ }^{2}$ Reactions were performed in singlet and consisted of $50 \mu \mathrm{l}$ compound at indicated concentrations, $75 \mu \mathrm{l}\left[{ }^{125} \mathrm{I}\right]-\mathrm{Tyr}^{13} \mathrm{MCH}(\sim 300 \mathrm{pM}, 2,200 \mathrm{Ci} / \mathrm{mmol}$; PerkinElmer Life Sciences, Boston, MA), and $75 \mu \mathrm{l}$ membrane protein (5-8 $\mu \mathrm{g})$ added sequentially in assay buffer to low binding 96-well plates (Corning, Palo Alto, CA). The reaction was allowed to reach equilibrium by incubation at room temperature for $30 \mathrm{~min}$. The amount of bound radioligand was determined by harvesting membranes via filtration through a UniFilter GF/C filter plate (PerkinElmer) using a UniFilter-96 vacuum manifold (PerkinElmer) (filters were pretreated with $1 \%$ polyethylenimine), washing once with 
$400 \mu \mathrm{l}$ wash buffer (PBS/ $0.01 \%$ Tween-20, pH 7.4), and measuring radioactivity by liquid scintillation using a TopCount NXT (PerkinElmer). Ki values were calculated using the Cheng-Prussoff equation using $\mathrm{K}_{\mathrm{d}}$ values of $0.2 \mathrm{nM}$ for $\left[{ }^{125} \mathrm{I}\right]-\mathrm{Tyr}^{13} \mathrm{MCH}$ as determined from saturation binding experiments.

Feeding Studies. Male Sprague-Dawley rats (3 weeks of age; Charles River LaboratorieS) were housed in triplicate in shoebox cages with corncob bedding and allowed ad libitum access to a medium high fat (32\%) diet (Research Diets, D12266B) for 12 weeks to induce obesity. After 12 weeks of feeding, diet-induced obese (DIO) rats were housed one per cage in hanging wire-bottom cages. Once per day, for 8 days prior to the beginning of the treatment period, animals were habituated to handling and oral gavage dosing with water. Baseline food intake and body weight were measured over this period. DIO rats $(553.63 \pm 22.89)$ were balanced for body weight and divided into 4 groups. During the hour before lights out, food, spillage and body weight were measured and animals were dosed with the vehicle, $0.25 \%$ methylcellulose $(n=7)$, or NBI-845 at 1 $\mathrm{mg} / \mathrm{kg}(\mathrm{n}=8), 3 \mathrm{mg} / \mathrm{kg}(\mathrm{n}=10)$, or $10 \mathrm{mg} / \mathrm{kg}(\mathrm{n}=10)$. Food intake and change in body weight from day 0 were analyzed by 2-way (treatment by day) mixed design analyses of variance (ANOVA). Holm-Sidak post hoc comparisons (verses the control group) were used to determine group differences.

Human Liver Microsome Clearance. Pooled human liver microsomes $(0.5 \mathrm{mg} / \mathrm{mL}$; $\mathrm{n}>10$; mixed gender) were incubated at $37^{\circ} \mathrm{C}$ with the compound in the presence of an NADPH-generating system containing $50 \mathrm{mM}, \mathrm{pH} 7.4$ potassium phosphate buffer, 3 mM magnesium chloride, $1 \mathrm{mM}$ EDTA, $1 \mathrm{mM}$ NADP, $5 \mathrm{mM}$ G-6-P, and $1 \mathrm{Unit} / \mathrm{mL}$ G-6PD. Incubations were conducted in six modified 2.0-mL, 96-well, deep-well plates in 1 $\mu \mathrm{M}$ of each compound ( $0.01 \%$ DMSO) with a total volume of $250 \mu \mathrm{l}$. Each plate, representing a single time point, contained 96 Titertube ${ }^{\circledR}$ Micro Tubes allowing for duplicates of 48 compounds at each time point $(0,5,10,20,40$, and 60 minuteS). Reaction was stopped by the addition of an appropriate stop reagent $(0.3 \mathrm{~mL}$ of acetonitrile containing a proprietary internal standard). Precipitated proteins were removed by centrifugation for $15 \mathrm{~min}$ at $3000 \mathrm{rpm}$, and the supernatant fluid $(\sim 0.1 \mathrm{~mL})$ 
was analyzed by LC/MS for the amount of parent compound remaining. The in vitro initial rates of metabolism were scaled using microsomal protein/gm liver, gm liver $/ \mathrm{kg}$ body weight and liver blood flow, to predict systemic clearance and \% bioavailability.

Rat Pharmacokinetics. The pharmacokinetics and blood brain barrier (BBB) penetrations were determined in male Sprague-Dawley rats following an intravenous (iv, $\mathrm{N}=3 /$ time point) and oral (po, $\mathrm{N}=3 /$ time point) dose. The dosing solution was prepared in purified water and filtered through a $0.2 \mu \mathrm{m}$ Nylon filter before administration $(2 \mathrm{~mL} / \mathrm{kg})$ via tail vein (iv) or a gavage (po). Blood and brain tissue samples were taken at predetermined time for composite sampling. All plasma and tissue samples were flash frozen in liquid nitrogen within 10 minutes of sampling and stored in $-70^{\circ} \mathrm{C}$ or below until analysis. The bioanalytical method applied for the measurement of test articles in plasma along with added internal standard consisted of precipitation with $200 \mu \mathrm{L}$ of acetonitrile from $50 \mu \mathrm{L}$ of plasma, centrifugation and recovery of the supernatant, drying down in vacuum then reconstitution in acetonitrile-water solutions before introducing into an LC-MS/MS system for analysis. The lower limit of quantification (LLOQ) for the analytical methods was $5 \mathrm{ng} / \mathrm{mL}$ of test article in plasma. The bioanalytical method applied for the measurement of test articles in brain tissue along with added internal standard consisted of homogenizing half of the brain tissue (longitude cut) in $2 \mathrm{~mL}$ of acetonitrile/water (50:50), centrifugation and recovery of the supernatant before introducing into an LC-MS/MS system for analysis. The lower limit of quantification (LLOQ) for the analytical methods was $5 \mathrm{ng} / \mathrm{g}$ of test article in brain tissue. All pharmacokinetic parameters were calculated from a non-compartmental model in WinNonlin program. Brain to plasma ratio was obtained by comparing brain AUC to plasma AUC.

\section{Supporting Information References}

1. Bryant, R. D.; Kunng, F.-A.; South, M. S. A large scale preparation of 3-chloro-5methoxypyridazine. J. Heterocycl. Chem. 1995, 32, 1473-1476. 
2. Ma, C.; Cho, S.; Falck, J. R.; Shin D. Synthetic studies on isoquinoline derivatives with multidrug resistance (MDR) modulating activity. Heterocycles 2004, 63, 75-85.

3. Hoare, S. R. J.; Sullivan, S. K.; Ling, N.; Crowe, P. D.; Grigoriadis, D. E. Mechanism of corticotropin-releasing factor type I receptor regulation by nonpeptide antagonists. Mol. Pharmacol. 2003, 63, 751-765. 